Forum $2021 \cdot 36: 355-356$

https://doi.org/10.1007/s12312-021-00985-2

Online publiziert: 1. Oktober 2021

(c) Springer Medizin Verlag $\mathrm{GmbH}$, ein Teil von

Springer Nature 2021

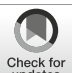

Check for
updates

\section{Neue Legislaturperiode: Neujustierung im Gesundheitswesen}

\author{
Thomas Seufferlein \\ Klinik für Innere Medizin I, Universitätsklinikum Ulm, Ulm, Deutschland
}
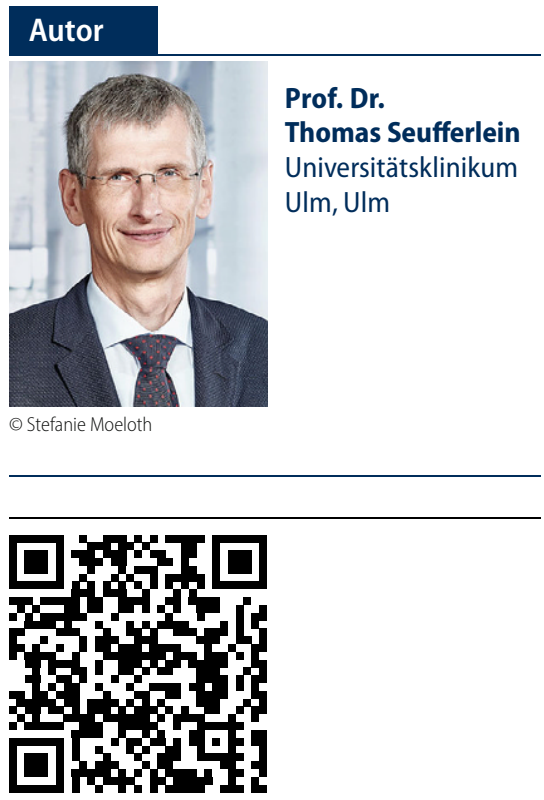

QR-Code scannen \& Beitrag online lesen
Wenn dieses Heft erscheint, ist der neue Bundestag gewählt und die Koalitionsverhandlungen haben vermutlich begonnen. Zu den Topgesundheitsthemen der neuen Legislaturperiode wird noch immer die COVID-19-Pandemie zählen. Dazu kommen neue Fragen, etwa die nach den gesundheitlichen Auswirkungen des Klimawandels, die auch bei uns inzwischen unmittelbar spürbar sind. Darüber hinaus blieb in der letzten Legislaturperiode vieles liegen. Vor allem im Bereich der Digitalisierung hinken wir im internationalen Vergleich hinterher. Das liegt u. a. am Investitionsstau, der sich über lange Zeit aufgebaut hat, weil Länder und Kommunen den Krankenhäusern jedes Jahr mehrere Milliarden Euro schuldig bleiben. Im stationären Bereich leidet die Krankenversorgung außerdem an einem Überangebot an Häusern in Ballungszentren, einer Versorgungslücke auf dem Land und einem nach wie vor großen Mangel an Pflegekräften.

Der finanzielle Druck, der allein durch die COVID-19-Pandemie für Staat, Bürger*innen und unser Gesundheitssystem entsteht, ist noch nicht in allen Einzelheiten absehbar. De facto verschlechtert sich aber derzeit die Lage der gesetzlichen Krankenkassen. Die Allgemeinen Ortskrankenkassen melden für das erste Halbjahr ein Defizit von 1,6 Mrd. $€$. Da sich laut AOK-Gemeinschaft für die zweite Jahreshälfte eine weitere Kostensteigerung abzeichnet, rechnet sie bis Jahresende mit einem Defizit von weit mehr als 4 Mrd. $€$.

Für die Krebsmedizin hat es in der vergangenen Legislaturperiode durchaus positive Entwicklungen gegeben: 2019 fiel der Startschuss für die vom Bundesministerium für Forschung und Bildung sowie vom Bundesgesundheitsministe- rium initiierte Nationale Dekade gegen Krebs mit dem Ziel, die Krebsforschung zu stärken. Das Gesetz zur Zusammenführung von Krebsregisterdaten soll künftig dafür sorgen, dass Informationen aus den Landeskrebsregistern zentral gebündelt und damit auch für die Versorgungsforschung besser zugänglich werden. Mit den Zentrumsregelungen des Gemeinen Bundesausschusses und den Verträgen für die integrierte Versorgung nach § 140 SGB V wurden Finanzierungsmöglichkeiten für den zusätzlichen Aufwand bei einer qualitativ hochwertigen Krebsversorgung an den von der Deutschen Krebsgesellschaft zertifizierten Zentren geschaffen.

Auch bei der bereits im Nationalen Krebsplan geforderte Regelfinanzierung der ambulanten Krebsberatungsstellen sind wir einen Schritt weitergekommen. Dank des Gesundheitsversorgungsweiterentwicklungsgesetzes erstatten Kassen rückwirkend zum Januar $202180 \%$ der Kosten für psychosoziale Beratungen; $15 \%$ der Kosten sollen über die Länder gedeckt und $5 \%$ durch Eigenmittel der Beratungsstellen getragen werden. Ein Schritt in die richtige Richtung, der jetzt allerdings durch die Qualitätskriterien, die der Spitzenverband der gesetzlichen Krankenkassen für die Förderung voraussetzt, möglicherweise an Wirkung verliert. Denn die Fördergrundsätze gehen nur auf einen Teil des Leistungsspektrums von Krebsberatungsstellen als niederschwellige regionale Anlaufstelle für Ratsuchende ein. Dabei sollen künftig auch Klinikbetreiber als Träger der ambulanten Krebsberatung infrage kommen. Das ist sicher nur dann sinnvoll, wenn sie ihre Beratungsstellen im Arbeitsablauf klar strukturell und räumlich von stationären 
Diensten trennen und für alle potenziell Ratsuchenden einer Region offenhalten. Ohne solche klaren Regelungen stellt sich die Frage, ob das neue Gesetz wirklich eine bessere Beratungssituation für die Betroffenen schafft - oder vielmehr (Fehl)Anreize setzt, um die Krebsberatung zur besseren Bindung der Patient*innen an das eigene Krankenhaus zu nutzen.

Ich wünsche mir von der Politik in der kommenden Legislaturperiode den Mut, Versorgungsthemen grundsätzlich anzugehen und dabei die Qualität und den Patientennutzen im Blick zu haben. 500.000 Krebsneuerkrankungen jährlich sind keine Kleinigkeit für ein Gesundheitswesen. Wir brauchen auch weiterhin die Förderung der Krebsforschung und Maßnahmen zur Wissen generierenden onkologischen Versorgung, wie sie gerade in Rahmen der Nationalen Dekade gegen Krebs angedacht und entwickelt werden. Die begonnenen Ansätze sollten keinem Sparzwang zum Opfer fallen.

\section{Korrespondenzadresse}

Prof. Dr. Thomas Seufferlein

Klinik für Innere Medizin I, Universitätsklinikum Ulm

Albert-Einstein-Allee 23, 89081 Ulm,

Deutschland

thomas.seufferlein@uniklinik-ulm.de

Interessenkonflikt. T. Seufferlein gibt an, dass kein Interessenkonflikt besteht.

\section{Krebs im Alter}

Standardisierte Tests können Überleben und Lebensqualität verbessern

Von Tumorerkrankungen sind vor allem ältere und hochbetagte Menschen betroffen. Neben dem Krebs kommen altersbedingt oft funktionale, kognitive und psychische Belastungen und Störungen erschwerend hinzu. Aber alt ist nicht gleich alt. Die Einschränkungen sind sehr unterschiedlich gelagert und ausgeprägt. Für die Ermittlung der Gesamtkonstitution stehen spezifische Tests für ältere Patientinnen und Patienten zur Verfügung. Sie unterstützen den Arzt, die Krebstherapie gezielt auf die individuelle Situation des Betroffenen zuzuschneiden. Krebspatienten ab 70 Jahren sollten sich bei den behandelnden Ärzten nach der Möglichkeit einer solchen Testung erkundigen.

Der menschliche Alterungsprozess ist komplex. Wie schnell und in welcher Form Menschen altern, ist individuell sehr unterschiedlich. Auch die Fähigkeit, altersbedingte Einschränkungen zu kompensieren, kann von Mensch zu Mensch anders sein. Geriatrische Testverfahren für ältere Patientinnen und Patienten helfen bei der Beurteilung der körperlichen und psychischen Verfassung und decken altersbedingte Probleme auf.

\section{Bessere Behandlungsplanung}

Körperlich und geistig fitte ältere Patienten vertragen die Krebstherapie in der Regel besser und leben länger als gebrechliche Patienten. Deshalb ist es wichtig, neben den Besonderheiten des Tumors bei der Ausrichtung der Therapie auch den allgemeinen Gesundheitszustand zu berücksichtigen. Wissenschaftliche Daten zeigen: Standardisierte Tests für Ältere führen bei rund einem Viertel der älteren Krebspatienten zu einer Änderung der Dosis, des Therapieintervalls oder der Therapieform. Wegen des nachweislich positiven Effekts auf Überleben und Lebensqualität wurden sie unter dem Fachbegriff "geriatrisches Assessment" in die onkologischen Leitlinien aufgenommen. Trotz dieser Empfehlungen werden sie allerdings noch zu selten eingesetzt.

\section{Was wird getestet?}

Zunächst erfolgt eine Art Schnelltest, auch als geriatrisches Screening bezeichnet. Abgefragt werden Nahrungsaufnahme und Gewichtsverlust in den letzten drei Monaten, Body Mass Index, Alter, die Fähigkeit vom Bett oder Stuhl aufzustehen und nach draußen zu gehen. Es wird dokumentiert, wie viele Medikamente der Patient einnimmt und ob psychologische Probleme vorliegen. Außerdem wird er gebeten, seinen Zustand - im Vergleich zu Gleichaltrigen - selbst einzuschätzen. Anhand des Ergebnisses wird entschieden, ob eine detaillierte AltersDiagnostik benötigt wird. Ist dies der Fall, werden weitere Tests durchgeführt. Sie beurteilen zum Beispiel die Bewältigung des Alltags, also etwa Ankleiden, Telefonieren oder das Regeln von Bankgeschäften. Weitere Themen sind körperliche, geistige und psychische Gesundheit sowie das soziale Umfeld.

\section{Vorteile für den Patienten?}

Krebsspezifische Behandlungen wie Operationen, Chemotherapie, Immun- und Strahlentherapie belasten ältere Patienten und Patientinnen in besonderem Maße. Mithilfe der Tests lässt sich abschätzen, über welche "persönlichen Reserven“ Krebspatienten im Alter verfügen, um eine geplante Krebsbehandlung erfolgreich zu bewältigen. Je nach individuellem Zustand kann die Therapie bestmöglich angepasst, Über- oder Untertherapie vermieden werden. Auch die Kommunikation zwischen Arzt und Patient profitiert von den Tests: Bei der gemeinsamen Behandlungsplanung können altersbedingte Probleme, aber auch persönliche Werte und Ziele offen benannt werden. Das schafft Vertrauen und gibt Raum für Fragen etwa zu Heilbarkeit, Lebenserwartung und Lebensqualität.

Weitere Informationen:

https://www.krebsinformationsdienst.de

Quelle: PM Deutsches

Krebsforschungszentrum, 21.09.2020 